has allowed us to develop the service in order to increase capacity, reduce pressures on unplanned care and reduce costs. Method 12 weeks of prospective data were collected on patients receiving elective paracentesis. Recommendations made on analysis of this data (Excel) led to changes in the standard operating procedure and a further 6 weeks of prospective data collection closed the PDSA cycle.

Results In the initial data collection, 31 elective paracentesis' were completed on 15 individual patients. Drains were in situ for a median of 24 hours, necessitating an overnight stay. On average $10,966 \mathrm{ml}$ of fluid were drained (range $4324 \mathrm{ml} \mathrm{-}$ $19692 \mathrm{ml})$. The rate of ascitic drainage plateaued at 8 hours, with an average, 74\% (range 53\%-100\%) of total ascitic fluid drained at this point. From this we hypothesized that adequate drainage could be achieved with a day case procedure.

During the second data collection, 27 elective paracentesis' were completed on 12 individual patients with drains in situ for 8 hours. On average $8,123 \mathrm{ml}$ of fluid were drained (range $3903 \mathrm{ml}-20996 \mathrm{ml}$ ). If we extrapolate this data over 12 weeks we could accommodate a $74 \%$ increase in capacity between study groups.

$20 \%$ albumin replaced $4.5 \%$ to reduce volume infused, therefore reducing further time constraints on admission. On average 3 bottles of $4.5 \%$ albumin were used in the initial study group and only 2 bottles of $20 \%$ in the second study group. This lead to a cost saving, on average of $£ 57.06$ per paracentesis, this becomes substantial when added to the cost savings of day case versus overnight stay.

There were no clinically significant changes in renal function or haemodynamic parameters in either data set. $86 \%$ of patients initially studied had a UKELD score greater than 49 with a median value of 54.5 (range 4-0).

There were 7 repeat attenders in the initial group and 4 in the second, attending 3 and 4 weekly, respectively. Only one patient was studied in both data sets with increased drain frequency from monthly to fortnightly.

Conclusions Both data sets have shown variability of ascitic fluid volume and drainage rates. We must continue an individualised approach to refractory ascites, but changes to our standard operating procedure have increased elective paracentesis capacity by $74 \%$, reduced pressure on unplanned care and reduced costs by $£ 793.06$ per paracentesis. The changes are safe with no clinically significant renal function or haemodynamic deterioration. It is difficult to attribute cause to changes in paracentesis frequency given the multiple confounding factors related to ascites production.

\section{PTU-089 HEPATITIS B SCREENING PRIOR TO RITUXIMAB AND SUBSEQUENT MANAGEMENT TO REDUCE THE RISK OF REACTIVATION}

${ }^{1}$ Grace Dolman*, ${ }^{2}$ loannis Theocharopoulos, 'Patrick Kennedy. 'Barts Liver Centre, London, UK; ${ }^{2}$ Barts and the London School of Medicine and Dentistry, London, UK

\subsection{6/gutjpl-2019-BSGAbstracts.448}

Introduction Hepatitis B (HBV) reactivation can occur during immune suppression in patients with serological evidence of current infection (HBsAg positive) or past exposure to $\mathrm{HBV}$ (HBsAg negative, anti-HBc positive). Rituximab poses a particular risk, with reported rates of reactivation $>10 \%$ in those with positive HBV markers. Reactivation can result in delays to ongoing treatment and, in small numbers of cases, acute liver failure leading to transplantation or death.

Methods We performed a retrospective audit of screening for HBV markers prior to rituximab and management of patients at risk of $\mathrm{HBV}$ reactivation in an East London NHS Trust serving a multi-ethnic population. Our cohort was identified from a search of electronic records for a pharmacy order for rituximab placed in 201-018 across clinical specialties.

Results 461 patients were included, of whom 191 were male (41\%). Ethnicity was as follows: British or Irish 169 (36.6\%); Asian or Asian British 118 (25.6\%); Black or Black British 44 (9.5\%); Any other white background 44 (9.5\%); Mixed 4 (0.9\%); Chinese 1 (0.2\%); Any other ethnic group 22 (4.8\%); Not known 59 (12.8\%). Screening was adequate in 384 patients (83.3\%). 62 patients (13.4\%) had undetectable HBsAg, but no record of anti-HBc. 2 patients (0.4\%) were not tested for $\mathrm{HBsAg}$, but were anti-HBc negative, making past/current infection unlikely. 13 patients (2.8\%) had no record of either $\mathrm{HBsAg}$ or anti-HBc testing. 339 patients (72.7\%) had undetectable HBsAg and anti-HBc. 3 patients $(0.7 \%)$ tested positive for $\mathrm{HBsAg}$, all of whom received appropriate antiviral prophylaxis. 42 patients (9.1\%) tested positive for anti-HBc, with undetectable $\mathrm{HBs}$. able that passive transmission had occurred as a result of immunoglobulin infusions in 2 cases and was confirmed in 3 cases. Repeat anti-HBc was pending in 2 patients. 5 of these 7 patients received antiviral prophylaxis. Anti-HBc positivity was thought to be due to past HBV exposure in 35 patients (7.6\%). One patient was already on Truvada for HIV infection. A further 27 patients received antiviral prophylaxis with either lamivudine, entecavir or tenofovir, although in 7 of these the prophylaxis commenced after rituximab infusions had started. No prophylaxis was given to 8 patients at risk of HBV reactivation based on serological markers $(21 \%$ of at risk group). There were no episodes of reactivation during the audit period.

Conclusion In this audit of a multi-ethnic population receiving rituximab, we found that screening was adequate in only $83 \%$ of cases. Of those adequately screened, nearly 10\% were at risk of hepatitis $\mathrm{B}$ reactivation with B-cell depleting therapies. $21 \%$ of those at risk did not receive appropriate prophylaxis. We propose cross-specialty guidelines and safety checkpoints in pharmacy and infusion units to reduce the risk of HBV reactivation in this patient group.

\section{PTU-090 THE USE OF FAECAL CALPROTECTIN AS A SCREENING TOOL FOR REFERRING PATIENTS WITH POSSIBLE IBD}

Darren Fernandes*, Aarati Mathew, David Elphick. Chesterfield Royal Hospital, Chesterfield, UK

10.1136/gutjnl-2019-BSGAbstracts.449

Introduction Faecal calprotectin (FC) has been shown to have high sensitivity and specificity for differentiating between Inflammatory Bowel Disease (IBD) and functional gastrointestinal disorders. However, there are different thresholds for interpreting FC results. The National Institute for Health and Care Excellence (NICE) therefore proposed the role of an intermediate range for values with Turvill et al. suggesting a cut off of $>100$. At Chesterfield Royal Hospital, no such intermediate range has been determined. The purpose of this study was thus to analyse FC results and referral outcomes to 